\title{
Inhibition of Dicing of Guanosine-Rich shRNAs by Quadruplex-Binding Compounds
}

\author{
Anja Henn ${ }_{1}{ }^{[a]}$ Astrid Joachimi, ${ }^{[a]}$ Diana P. N. Gonçalves, ${ }^{[b]}$ David Monchaud ${ }^{\left[{ }^{[c]}\right.}$ \\ Marie-Paule Teulade-Fichou, ${ }^{[c]}$ Jeremy K. M. Sanders, ${ }^{[b]}$ and Jörg S. Hartig ${ }^{*[a]}$
}

\begin{abstract}
RNA interference is triggered by small hairpin precursors that are processed by the endonuclease dicer to yield active species such as siRNAs and miRNAs. To regulate the RNAi-mediated suppression of gene expression, we imagined a strategy that relies on the sequence-specific inhibition of shRNA precursor processing by immediate RNA-small molecule interactions. Here, we present a first step in this direction by augmenting shRNAs with guanosinerich sequences that are prone to fold into four-stranded structures. The addition of small molecules that selectively bind to such quadruplex sequences should allow for the specific inhibition of dicing of shRNAs that contain suitable G-rich elements. In an attempt to find compounds that protect against dicer proc-
\end{abstract}

essing, we have examined the effects of quadruplex-binding compounds on the dicer processing of shRNAs containing G-quadruplexes. Although a variety of small molecules that are known to bind to quadruplexes inhibited in vitro dicing of shRNAs, only two substance classes, namely certain porphyrazines and bisquinolinium compounds, showed selective inhibition of G-rich shRNAs compared to control sequences lacking guanine-rich elements. The G-rich shRNAs displayed a potent knockdown of gene expression in mammalian cell culture, but the effect was not influenced by addition of the respective quadruplex-binding compounds.

\section{Introduction}

One of the most interesting tasks of modern chemical biology is the quest for compounds that enable the external regulation of key processes in cells. The recently discovered functional RNAs represent valuable targets for such interventions. RNA interference (RNAi) describes a phenomenon that is common to higher organisms; small double-stranded RNAs act as triggers that ultimately result in the suppression of gene expression by several related mechanisms. ${ }^{[1,2]}$ Within the RNAi pathway, genetically encoded microRNA (miRNA) precursors are transcribed as pri-miRNAs and processed by Drosha to yield premiRNAs. ${ }^{[3]}$ These precursors are composed of short hairpin RNAs (shRNAs), which are then exported to the cytoplasm where they are further processed by the endonuclease dicer to yield $\sim 21 \mathrm{nt}$ double-stranded miRNAs or siRNAs (short interfering RNAs). ${ }^{[4-6]}$ The short RNAs are subsequently assembled into the RNA-induced silencing complex (RISC). ${ }^{[7]}$ Ultimately, only one strand remains in the complex, which then serves as a guide to hybridize to a complementary target transcript. ${ }^{[8]}$ Suppression of gene expression can take place by related mechanisms such as Argonaute-mediated cleavage of the target RNA or suppression of translation upon binding of the guided RISC to the $3^{\prime}$-untranslated region of the target mRNA. ${ }^{[9]}$ Chromatin remodelling can also be guided through the RNAi pathway. ${ }^{[10]}$ Externally applied siRNAs enter the RISC complex immediately and hence allow the control of gene expression through the addition of synthetic RNAs. ${ }^{[1]]}$ In recent years, species consisting of small hairpin precursors (shRNAs) have been applied that often display advantages compared to siRNAs consisting of two short $21 \mathrm{nt}$ RNAs. These shRNAs are processed by dicer as well, and often display higher knockdown activities com- pared to siRNA sequences. ${ }^{[12]}$ In addition to achieving potent and reliable knockdown of gene expression by using the RNAi pathway, it would be very helpful to gain external temporal control over the knockdown activity of these RNA species. For example, the development of tools that allow us to directly switch on or off the knockdown activity of such interfering RNAs by immediate interaction of a shRNA-specific compound would be a major advancement.

Compounds that bind selectively to naturally occurring RNA motifs are highly desirable. Recently, compounds that bind specifically to certain hairpin structures have been introduced. ${ }^{[13,14]}$ After screening peptide libraries, Davies and Arenz ${ }^{[15]}$ identified a compound that was able to achieve control over dicer processing of shRNAs. In addition, naturally occurring, functional RNAs have been engineered to fulfil novel tasks. ${ }^{[16,17]}$ RNAs represent ideal tools to implement synthetic

[a] A. Henn, A. Joachimi, Prof. Dr. J. S. Hartig

Department of Chemistry and

Konstanz Research School Chemical Biology (KoRS-CB)

University of Konstanz

Universitätsstrasse 10, 78457 Konstanz (Germany)

Fax: (+49) 7531-885140

E-mail:joerg.hartig@uni-konstanz.de

[b] Dr. D. P. N. Gonçalves, Prof. Dr. J. K. M. Sanders University Chemical Laboratory, University of Cambridge Lensfield Road, Cambridge CB2 1EW (UK)

[c] D. Monchaud, Dr. M.-P. Teulade-Fichou Institut Curie, CNRS-UMR 176

Centre Universitaire Paris XI, 91405 Orsay (France) 
functions in cells because modular design strategies and programmable structure-function relationships can often be applied. For instance, several examples of artificial switches of gene expression have been constructed by implementing small molecule-dependent RNA modules in mRNAs. ${ }^{[18]}$ Switching of nucleic acid structure and function is often made possible by augmenting the respective RNA with sequence modules that can be addressed by small molecules. Aptamer sequences are ideally suited for such purposes. ${ }^{[19]}$ To achieve small molecule-regulated control of gene expression, Smolke and coworkers developed antisense constructs that can be triggered through an aptamer-small molecule interaction. ${ }^{[20]}$ For the in cis-regulation of gene expression, aptamers as well as aptazymes have been incorporated into mRNAs; this results in switchable elements for small molecule-dependent gene expression. ${ }^{[21,22]}$ With respect to RNA interference, one example is known in which attachment of an aptamer to a shRNA resulted in small molecule-dependent regulation of RNA interference. ${ }^{[23]}$

In addition to using aptamers as addressable domains in functional RNAs, we are interested in exploring the possibility of using four-stranded, guanosine-rich sequences for controlling nucleic acid functions. Such G-quadruplexes could be well suited as regulatory domains in nucleic acids because induction of a four-stranded structure results in substantial rearrangement of the respective nucleic acid. In addition, several compound classes have been described that interact with and stabilize quadruplexes; this opens the possibility of inducing structural rearrangements that could be exploited for controlling nucleic acid functions. ${ }^{[24-26]}$ We have successfully demonstrated the potential of quadruplexes as regulatory elements by using them to modulate the activity of a hammerhead ribozyme. ${ }^{[27]}$ In a second study, we have shown that the binding affinity of aptamers based on quadruplex structures can be controlled by quadruplex-binding ligands. ${ }^{[28]}$ Moreover, modulation of gene expression was realized upon insertion of G-quadruplexes into mRNAs. ${ }^{[29]}$ Here, we present a novel strategy for controlling the dicer-mediated processing of shRNAs by using quadruplex-selective ligands.

To establish a modular approach that would enable regulation of nucleic acid functions through a general mechanism, we investigated whether quadruplex-small molecule interactions are suitable switching devices. G-rich, four-stranded sequences can adopt a variety of topologies. ${ }^{[30]}$ The formation of quadruplex structures is suspected to play important roles in the regulation of genetic mechanisms. Most prominently, Grich DNA repeats that are prone to fold into quadruplexes are not only found in the telomeric regions of higher organisms, but are also abundant in certain promoter regions. ${ }^{[31-34]} \mathrm{Al}-$ though RNA quadruplexes have not yet been studied in much detail, there are some reports that hint at roles in the regulation of gene expression as well as RNA processing such as splicing. ${ }^{[29,35,36]}$ Significant advances in the field of selective recognition of quadruplexes have been made in recent years. Several compounds have been described that seem to act by recognition or induction of quadruplexes in cells. For example, a variety of compounds display antitumour activities by interacting with telomeres as well as G-rich promoter elements. ${ }^{[37-42]}$
With respect to interfering with quadruplex functions inside cells, the quest for compounds that discriminate between quadruplex, duplex, and single-stranded nucleic acids is especially important, and several examples have already been reported. ${ }^{[43,44]}$ It is even possible to use compounds to discriminate between the different naturally occurring quadruplex structures. ${ }^{[45,46]}$ With respect to the mechanism of action of such compounds, several possibilities such as stabilization of a quadruplex, induction, as well as chaperoning of the quadruplex fold need to be considered. ${ }^{[4]]}$

\section{Results and Discussion}

\section{Screening for dicer inhibition}

To find shRNAs that can be controlled by small molecule interactions, we searched for shRNA sequences that are correctly processed by dicer, but allow the inhibition of dicing upon addition of a suitable compound, see Figure $1 \mathrm{~A}$. We constructed shRNAs containing G-rich stretches that could, in principle, facilitate formation of quadruplex structures, see shRNA 5 in Figure $1 \mathrm{~B}$. To identify specific interactions, we also tested a second shRNA that lacks the G-rich stretches, see sequence shRNA 2 in Figure 1 B. In order to lower the hybridization energies, mutations of the passenger strand were introduced; this led to mismatched sequences. We anticipated that upon addition of the quadruplex-interacting compounds, it would be easier to interfere with the duplex fold, which is required for correct processing. The tested shRNA sequences were diced to the appropriate siRNAs; for an example of a dicing reaction see Figure 2 A. From both shRNA 2 as well as shRNA 5, 21$22 \mathrm{nt}$ siRNAs are generated, even though the precursor shRNAs have different lengths. The dicer product appears as a double band. Because the shRNAs were internally labelled during transcription, it is likely that the two major bands correspond to both strands of the mature siRNA. Having established that the designed shRNAs are correctly processed by dicer, we next tested a variety of small molecules that are known to bind to quadruplexes for their ability to interfere with the dicing process.

To screen for small molecules that inhibit the dicing reaction, compounds were tested in varying concentrations in combination with both shRNAs. Positive hits were identified by increased amounts of unprocessed precursor shRNA in combination with a reduction of the correctly diced $\sim 21-22 \mathrm{nt}$ siRNA. Particularly, specificity of interference with dicing was checked by comparing the compound's effects towards the G-rich shRNA (shRNA 5) with the respective control shRNA (shRNA 2). $A$ variety of compounds were screened for their ability to interfere with shRNA dicing: PIPER ( $N, N^{\prime}$-bis[2-(1-piperidino)ethyl]3,4,9,10-perylenetetracarboxylic diimide, a perylene derivative), ${ }^{[48]}$ 2,6-bis[3-(N-piperidino)propionamido]anthracene-9,10dione ${ }^{[49,50]} 3,6$-bis(3-piperidinopropionamido)acridine, ${ }^{[51,52]}$ cationic porphyrins such as TMPyP4 ${ }^{[53-56]}$ and mesoporphyrins. ${ }^{[55,57]}$ Most of the investigated compounds that are known to bind to quadruplex sequences potently inhibited dicer-mediated processing of the shRNAs ( $\mathrm{nm}$ to low $\mu \mathrm{m}$ concentrations, data 
A)

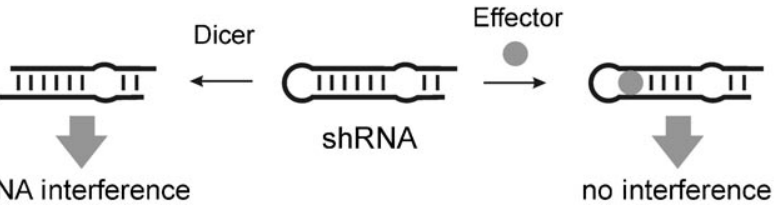

B)

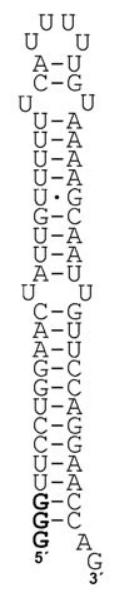

ShRNA 2

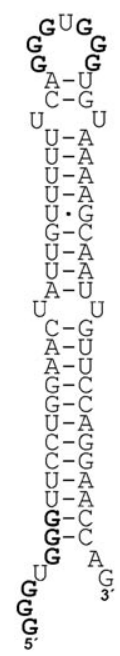

ShRNA 5

C)

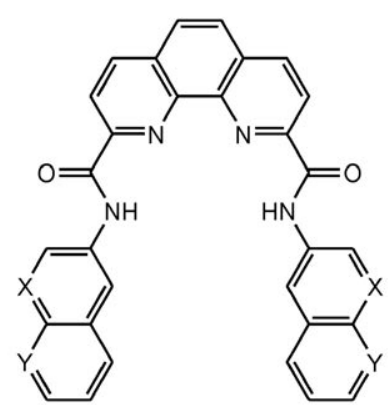

Phen-DC3: $\mathrm{X}=\mathrm{N}^{+} \mathrm{CH}_{3} \mathrm{Y}=\mathrm{CH}$ Phen-DC6: $X=\mathrm{CH} Y=\mathrm{N}^{+} \mathrm{CH}_{3}$

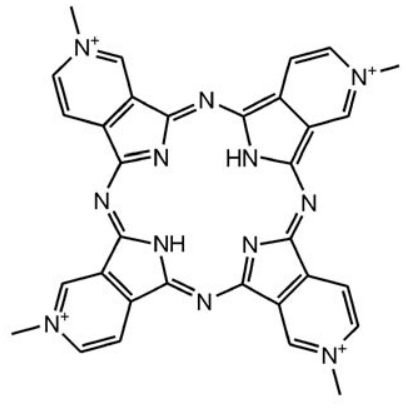

TmPyPz

TmPyPzZn (Zn-metallated)

Figure 1. A) Principle of small molecule-regulated dicing of shRNAs. shRNAs are processed by dicer to yield active, small interfering RNAs. Upon addition of suitable nucleic acid ligands, shRNA dicing is inhibited, which results in enhanced gene expression. B) shRNA constructs that were used in this study. C) Quadruplex-specific compounds that were used in this study (bisquinolinium compounds Phen-DC3 and Phen-DC6 as well as porphyrazines TmPyPz and TmPyPzZn).

not shown). This result could be expected because all of the tested compounds are known to bind to nucleic acids, and hence, have the potential to interfere with the dicing process. Nevertheless, in most cases dicing of the control sequences that lack G-rich stretches was inhibited to the same extent. This result hints at a rather unspecific binding mechanism that does not discriminate between G-rich and control sequences.

To selectively interfere with shRNA processing of G-rich sequences, we next tested certain compound classes that have been described recently to display selectivity towards quadruplex nucleic acids. Indeed, we were able to identify compounds, namely certain bisquinolinium compounds ${ }^{[43]}$ and porphyrazines $^{[44]}$ that were able to sequence-specifically inhibit dicing of a G-rich shRNA, while still allowing processing of the the control shRNA to occur. Specific inhibition of dicing depends on the respective design of the shRNA. Here, we focus on the sequence shRNA 5 and the corresponding control shRNA 2, shown in Figure 1B, which resulted in the highest selectivity of inhibition of dicing.

\section{In vitro characterization of compounds that interfere with shRNA dicing}

To compare the effectiveness of dicer processing of both shRNA 5 and shRNA 2 to yield the mature siRNAs, dicer cleavage kinetics were carried out at varying enzyme concentrations. As can be seen in Figure S2 in the Supporting Information, both RNAs are processed effectively, although shRNA 2 seems to be cleaved slightly more efficiently. The difference could originate from the less-preferential design of shRNA 5 . In addition to the $3^{\prime}$-overhang it contains a $5^{\prime}$-overhang, which could interfere with dicer recognition the shRNA. To better compare the compounds' effects on the dicing reaction, in the following experiments the dicer concentration was increased accordingly in reactions that contain shRNA 5 compared to shRNA 2. The effects of the identified porphyrazines and bisquinolinium compounds on the dicing reaction was then characterized by determining the initial rates of the dicer cleavage reaction in the presence of varying compound concentrations.

As can be seen in Figure 3, the bisquinolinium compound Phen-DC3 as well as the $\mathrm{Zn}$-complexed cationic porphyrin TMPyPzZn showed the ability to specifically inhibit the processing of G-rich over control shRNAs. The metal-free porphyrazine exhibits a reduced capability of discrimination between shRNA 5 and 2. The second bisquinoline that was tested (Phen-DC6) did not differentiate under these conditions. It must be noted that we have now characterized the effects of the compounds by measuring dicer cleavage rates instead of using end-point determinations of dicing reactions as was used before. In this respect, the observed selectivity of some of the tested compounds towards the G-rich sequence is very promising. For example, the compound with the highest selectivity for shRNA 5, Phen-DC3, shows complete inhibition of the cleavage reaction at $7.5 \mu \mathrm{M}$ whereas cleavage of shRNA 2 is almost unaffected. To evaluate whether the selectivity that was observed in the in vitro dicing reactions results from differen- 
A)

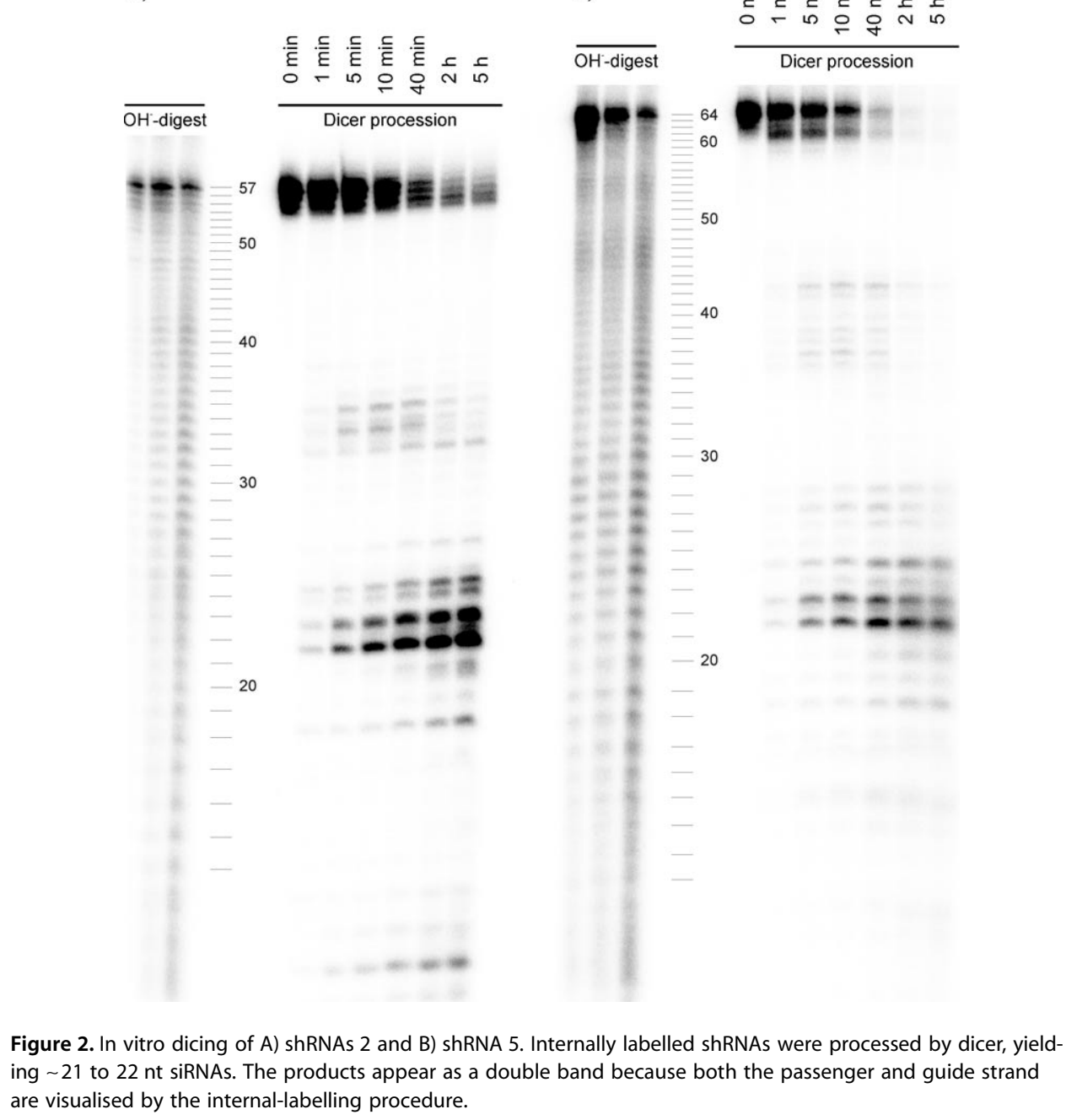

Figure 2. In vitro dicing of A) shRNAs 2 and B) shRNA 5. Internally labelled shRNAs were processed by dicer, yielding $\sim 21$ to $22 \mathrm{nt}$ siRNAs. The products appear as a double band because both the passenger and guide strand are visualised by the internal-labelling procedure.

B) binding site can be estimated by changes in cleavage intensities. More accurately, the changes in the self-cleavage pattern reflect changes in the flexibility of the respective RNA conformation.

As shown in Figure 4, addition of the quadruplex-interactive compounds did not significantly change the cleavage pattern of shRNA 2; this indicates that the compounds did not change the conformation of the shRNA. In contrast, shRNA 5 exhibited decreased cleavage of the loop sequences, especially in the presence of TmPyPzZn. This finding is in accordance with the hypothesis that the compounds preferentially bind to G-rich sequences. The probing experiments demonstrate that the flexibility of the loop structure of shRNA 5 is selectively reduced upon compound binding. These results encouraged us to test whether we could interfere with the dicing process in cell culture to selectively target RNAi-mediated knockdown of the G-rich shRNA by using the identified compound classes. ces in binding affinity of the respective compounds to the shRNAs, we carried out surface plasmon resonance studies. As shown in Table 1, the observed dissociation constants indeed reflect the behavior in the enzymatic studies; The discriminating compounds Phen-DC3 and TMPyPzZn show much higher affinity towards the G-rich shRNA 5 compared to the control shRNA 2. The less specific inhibition of dicing by Phen-DC6 and TMPyPz is in accordance with a smaller difference in binding affinity. Strikingly, the bisquinolinium compound, PhenDC3, that showed high discrimination in the enzymatic reactions also displayed strong selectivity in the binding experiments (see Figure S2 in the Supporting Information).

To investigate the structural basis of the differential recognition of the two shRNAs by the compounds, we carried out inline probing experiments. In-line probing is based on the spontaneous cleavage reaction of the phosphodiester bond by inline attack of the $2^{\prime}-\mathrm{OH}$ group and yields information on the structure and flexibility of the respective RNA sequences. For the two shRNAs, cleavage sites are mainly found in the loop regions, which is in accordance with the higher flexibility of these sites (see Figure 4). Upon addition of compounds, the

\section{Effects of shRNAs and compounds in human cell culture}

To characterize the effects of the designed shRNAs in combination with the identified compounds, we first tested the knockdown efficiency of the shRNAs in mammalian cell culture. For this purpose, the shRNAs were co-transfected into HEK cells together with constructs carrying firefly and renilla reporter genes. Both shRNAs target the same sequence of the firefly luciferase; renilla luciferase expression was used as an internal control. Both shRNAs showed very potent and specific knockdown of firefly luciferase expression, see Figure 5 . With both shRNAs, knockdown to less than $10 \%$ of residual expression was observed by using concentrations as low as $0.1 \mathrm{pmol}$ of shRNA in $500 \mu \mathrm{L}$ of cell culture medium ( $0.2 \mathrm{~nm})$. We observed, however, a slightly reduced activity of shRNA 5 compared to siRNA 2 at even lower concentrations. This result could originate from the reduced in vitro dicing efficiency that was observed with shRNA 5 . Next, we tested various compound concentrations with $0.1 \mathrm{pmol}(0.2 \mathrm{~nm})$ of added shRNA. The compounds were added two hours after the shRNA transfection procedure because simultaneous addition of transfection reagent and compounds showed toxic effects. Unfortunately, 
A)

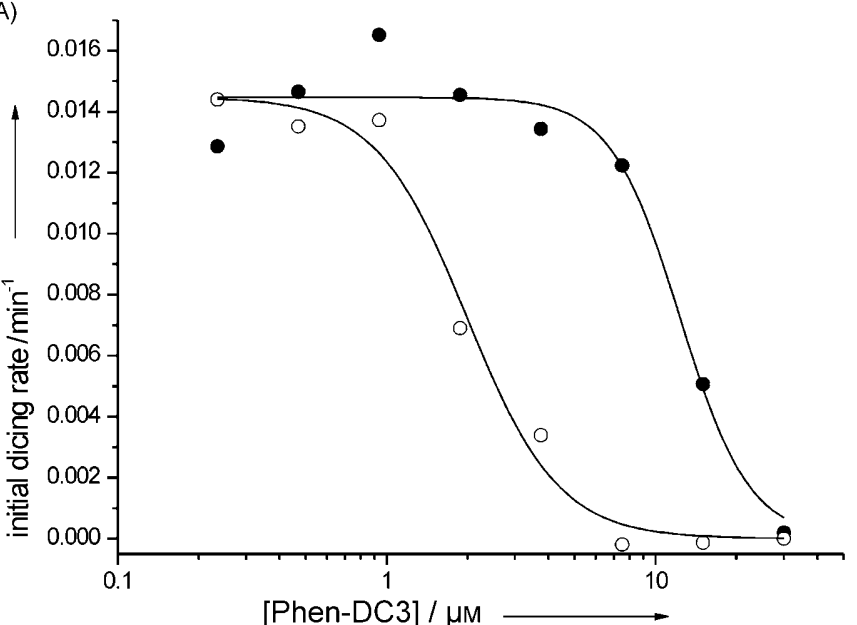

C)

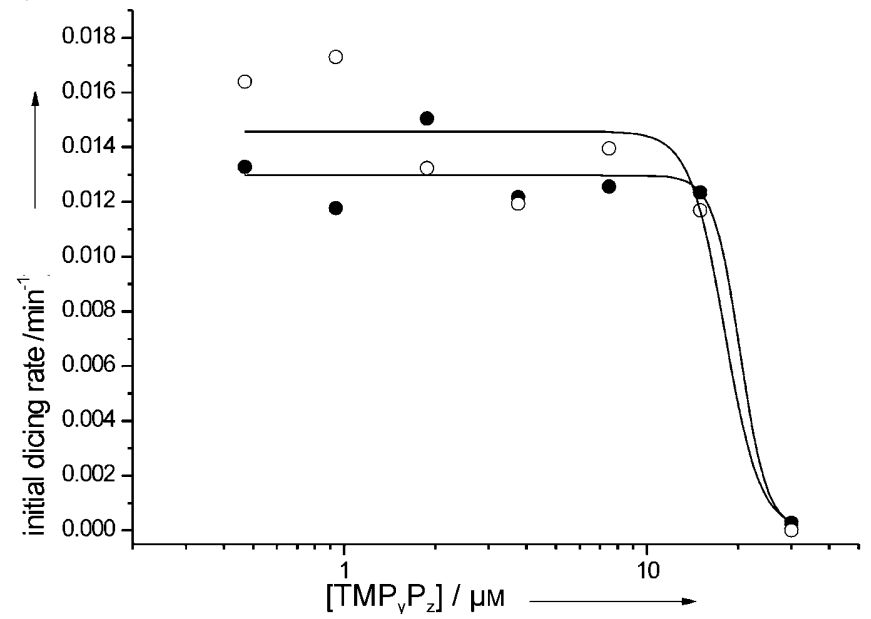

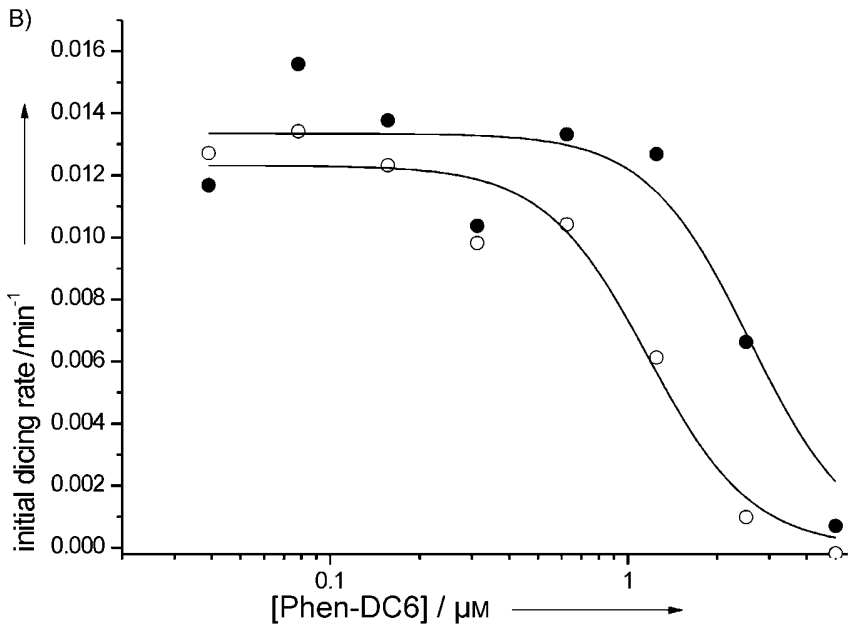

D)

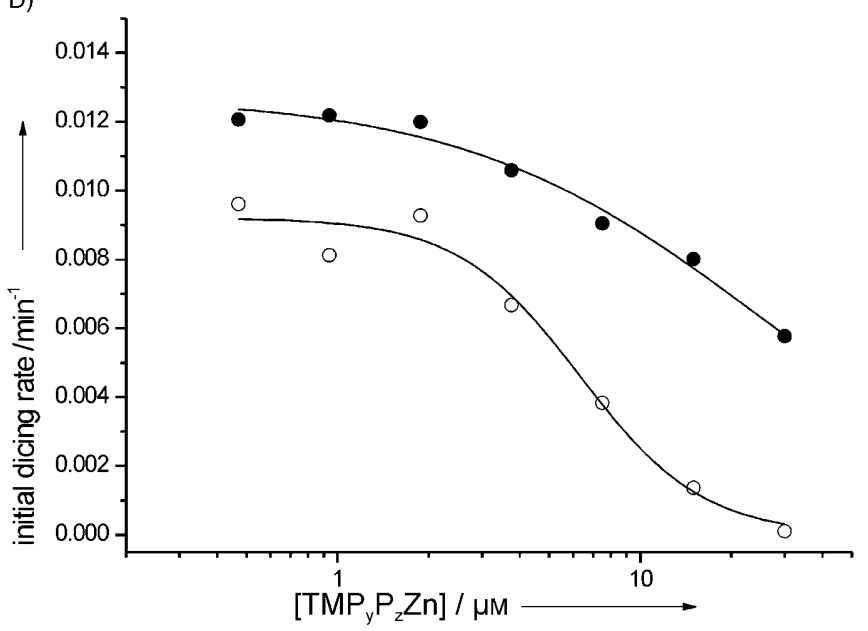

Figure 3. Small molecule-regulated dicing of shRNAs. Dicer processing kinetics in dependence to quadruplex-binding compounds. Data points represent initial cleavage rates. A) Phen-DC3; B) Phen-DC6; C) TMPyPz; D) TMPyPzZn; closed circles: shRNA 2; open circles: shRNA 5.

Table 1. Dissociation constants $\left(K_{\mathrm{d}}\right)$ in $\mathrm{nm}$ determined by surface plasmon resonance. For sensorgrams and fitting of dissociation constants see the Supporting Information.

\begin{tabular}{|lllll|} 
& Phen-DC3 & Phen-DC6 & TMPyPz & TMPyPzZn \\
\hline shRNA 2 & $-^{[a]}$ & 450 & 80 & 310 \\
shRNA 5 & 290 & 330 & 23 & 46
\end{tabular}

[a] No binding detected.

none of the compounds were able to reverse the knockdown effect. At higher concentrations, some of the compounds resulted in unspecific reduction of gene expression, which was observed by both decreased firefly and renilla luciferase expression (data not shown). This behavior might result of general, unspecificity, although the compounds did not display strong toxicity at these concentrations as determined by using a standard MTT assay (data not shown).

\section{Conclusions}

We have used quadruplex-specific compounds in combination with G-rich sequences attached to shRNAs to selectively control dicer-mediated shRNA cleavage. In the dicing reactions with purified enzyme and shRNAs, some of the tested compounds show promising discrimination between G-rich and control sequences. Although more dicer was used in the shRNA 5 dicing reactions, Phen-DC3 and TMPyPzZn were still able to selectively interfere with dicing of shRNA 5; much more pronounced dicing was observed with shRNA 2 at the same compound concentrations. Surface plasmon resonance studies demonstrated that the compounds indeed show increased binding affinity towards the G-rich sequence compared to the control shRNA. By using in-line probing we were able to demonstrate that the quadruplex-binding compounds selectively interfere with the G-rich loop of shRNA 5; however, we were not able to prove the formation of a quadruplex structure. One could speculate that compound binding to the G-rich shRNA induces an alternate structure such as a fourstranded quadruplex, which would prevent dicer from recognizing the RNA as substrate. It seems that the conformations 
A)

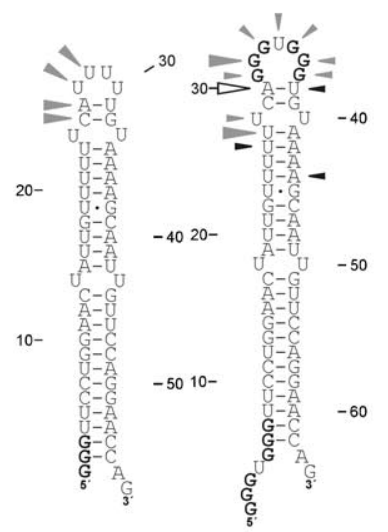

ShRNA 2

ShRNA 5
Phen-DC3

B)

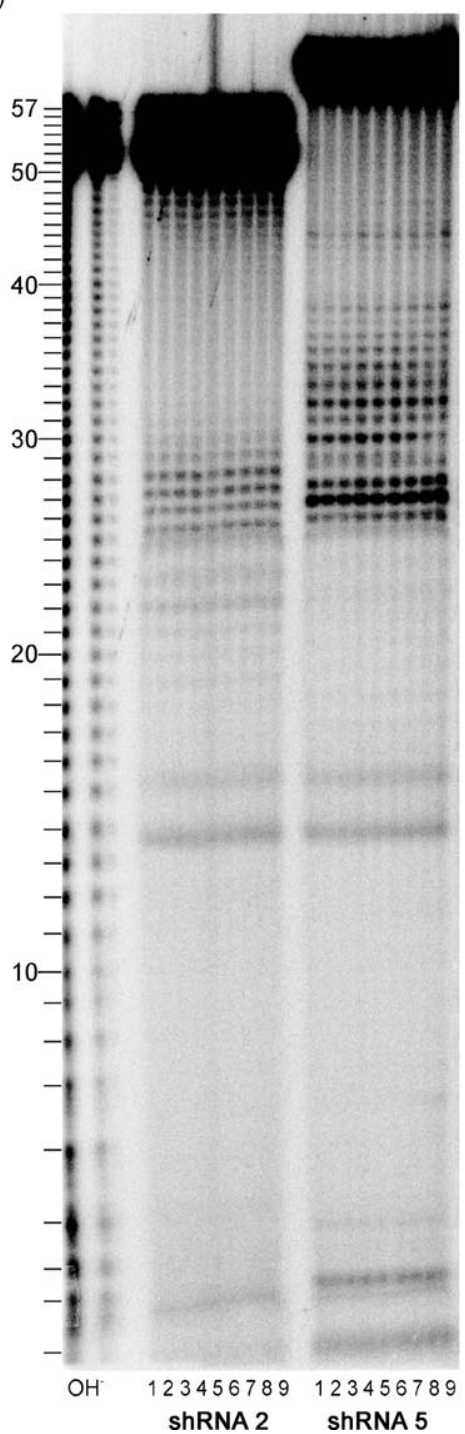

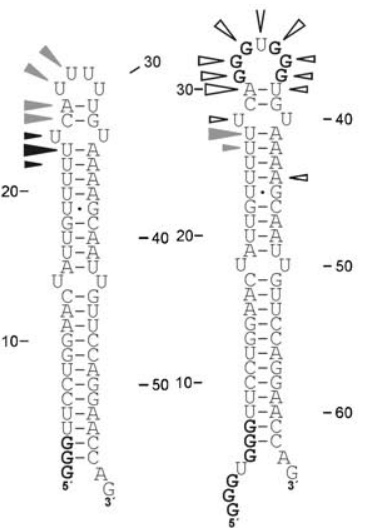

ShRNA 2

ShRNA 5

\section{TmPyPzZn}

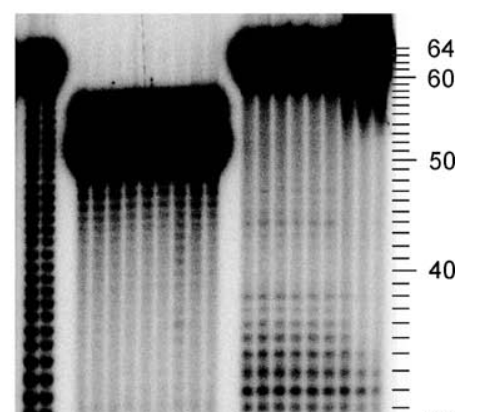

of the ribose and glycosidic bond generally force RNA quadruplexes into parallel topologies. ${ }^{[58]}$ Nevertheless, formation of a potential structure that is composed of a quadruplex with one strand of the shRNA residing in a loop that traverses the grooves of the quadruplex is unlikely because more dramatic changes of the probing data could be expected. Circular dichroism spectroscopy is not suited to clarify the structure of the compound-bound shRNA because both A-form RNA duplexes as well as paralleloriented quadruplexes show very similar CD spectra with a strong maximum around $265 \mathrm{~nm}$. More elaborate structural elucidation is necessary to address the exact mechanism of action.

When introduced into cells, shRNA 5 efficiently knocks down gene expression, although slightly less efficiently than shRNA 2 . The slightly reduced effects of the tested shRNAs in the cell culture experiments are in accordance with the observed in vitro dicing activity of shRNA 5 compared to shRNA 2 and could originate from the less-favorable design of the $G$ rich shRNA 5. Because dicer recognizes the two-nucleotide overhang at the $3^{\prime}$-end, ${ }^{[59]}$ the additional five nucleotides at the $5^{\prime}$-end of shRNA 5 might interfere with dicer recognition and hence provide a possible explanation for both the slightly reduced in vitro dicing efficiency as well as the slightly decreased in vivo knockdown of shRNA 5 . Upon addition of the compounds that were identified in the in vitro dicing reactions, we were not able to detect reversal of the shRNA-mediated knockdown of gene expression. Possible explanations for this observation could be that the compounds are not efficiently uptaken or that they bind unspecifically to other nucleic acids. Because the specificity of the compounds needs to be very high to discriminate between the quadruplex-forming shRNAs and the vast excess of cellular nucleic acids, even more selective ligands might be needed to specifically address G-rich sequences inside cells. Nevertheless, G-quadruplex-interacting compounds have been used before to interfere with nucleic acid functions within cells. For example, TMPyP4 has been shown to interact with telomeres as well as G-rich promoter elements. ${ }^{[38,60,61]}$ The potential problem of compounds that lack high specificity towards quadruplexes versus duplexes could be addressed by testing more specific compounds. As has been recently shown by Balasubramanian and co-workers, certain classes of triarylpyridines are able to discriminate even between different naturally occurring quadruplex sequences. ${ }^{[45,46]}$ With these promising advances of chemists working in the field of quadruplex-recognizing compounds, it might be possible to use quadruplex motifs as elements to control shRNAs as well as other interesting nucleic acid functions in the near future. 


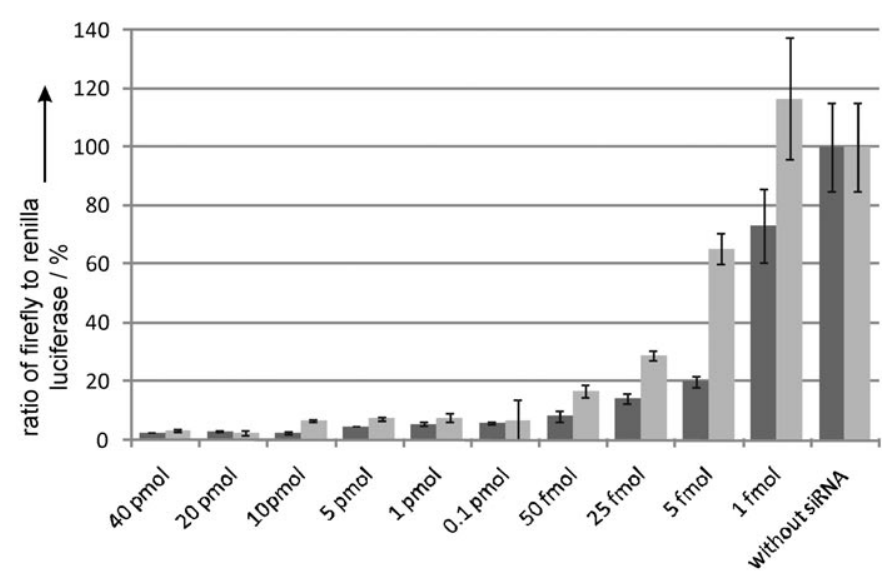

Figure 5. Effects of siRNA 2 and siRNA 5 in human cell culture. The indicated amount of siRNA was co-transfected with two plasmids that harbour firefly and renilla luciferase reporter genes in a total volume of $500 \mu \mathrm{L}$ medium (shRNA 2: dark gray; shRNA 5: light gray), and the luciferase activity was determined. The ratio of firefly/renilla activity is shown. Both shRNAs display potent knockdown of gene expression although shRNA 2 is slightly more active.

\section{Experimental Section}

shRNA transcription: DNA sequences that contained a T7 promoter sequence and encoded shRNAs were amplified by PCR. After precipitation with $\mathrm{EtOH}$, the whole PCR product was used for an in vitro transcription reaction. $\alpha{ }^{-32}$ P-ATP was used for internal labelling of the RNA. The transcription reaction was treated with DNAse I, and the RNA was purified by preparative PAGE.

Synthesis of quadruplex-binding compounds Phen-DC and TMPyPz: Phen-DC3 and Phen-DC6 as well as the porphyrazines TMPyPz and TMPyPzZn were prepared as described previously. ${ }^{[43,44]}$

In vitro dicing reactions: For the cleavage reaction of the shRNAs into siRNAs, we used the Recombinant Human Dicer Enzyme Kit from peqlab (Erlangen, Germany). Reactions contained the internally labelled RNA $(1 \mu \mathrm{g})$ in $1 \times$ Dicer Reaction Buffer $(20 \mu \mathrm{L})$, which consisted of ATP $(1 \mathrm{~mm})$ and $\mathrm{MgCl}_{2}(2,5 \mathrm{~mm})$. Compounds $(2 \mu \mathrm{L})$ of in different concentrations and $0.0125 \mathrm{U}$ dicer (when dsRNA 2 was used) or $0.0188 \mathrm{U}$ dicer (when dsRNA 5 was used) were incubated; this corresponded to approximately a 25 -fold excess of substrate. The reactions were incubated at $37^{\circ} \mathrm{C}$. After $2 \mathrm{~min}, 5 \mathrm{~min}, 10 \mathrm{~min}$, $30 \mathrm{~min}$ and $60 \mathrm{~min}$ aliquots $(2 \mu \mathrm{L})$ were sampled, and the reactions were stopped by adding PAGE loading buffer $(4 \mu \mathrm{L})$. The samples were analyzed by $10 \%$ denaturing PAGE and autoradiography. For the determination of initial dicer cleavage kinetics, the initial, linear phase of the reaction was fitted.

Surface plasmon resonance studies: The studies were carried out by using a Biacore T100 instrument (Uppsala, Sweden). For the determination of dissociation constants, each biotinylated shRNA (1000 RU) was immobilized on the surface of a streptavidin-derivatized sensor chip. The binding experiments were carried out by injecting various concentrations of the respective compounds. Dicing buffer was used as running as well as sample solution. The $K_{\mathrm{d}}$ values were determined by using the 1:1 binding model by fitting association and dissociation rates by using the Biacore $\mathrm{T} 100$ evaluation software.

In-line probing studies: Traces of $5^{\prime}-{ }^{32} \mathrm{P}$-labelled shRNAs were incubated in dicer buffer $(\mathrm{pH} 8.5)$ for three days at $25^{\circ} \mathrm{C}$ that contained varying amounts of compounds. The samples were analyzed by $10 \%$ denaturing PAGE and autoradiography.

Human cell culture and dual luciferase assay: HEK 293 cells were resuspended in DMEM + GlutaMAX-I medium (GIBCO ${ }^{\circledR}$ Invitrogen) supplemented with $10 \% \mathrm{FBS}$ and $1 \%$ penicillin/streptomycin (Sigma-Aldrich), seeded in 24-well plates with a final density of 50000 cells per well, and incubated at $37^{\circ} \mathrm{C}$ and $5 \% \mathrm{CO}_{2}$. Transfection of shRNAs and reporter plasmids were performed by using Lipofectamine ${ }^{\mathrm{TM}} 2000$ (Invitrogen). Cells were transfected with of pGL3 (firefly luciferase reporter construct, $125 \mathrm{ng}$ per well) and pRL (renilla luciferase control plasmid, $1.25 \mathrm{ng}$ per well, Promega Dual Luciferase Assay) and varying amounts of shRNAs. $2 \mathrm{~h}$ after transfection, the compounds were added and cells were incubated for another $24 \mathrm{~h}$ at $37^{\circ} \mathrm{C}$ and $5 \% \mathrm{CO}_{2}$. Gene expression was determined by using the Dual-Luciferase Reporter Assay System (Promega). The growth medium was removed, and the wells were washed by adding a sufficient volume of $1 \times$ PBS. $1 \times$ Passive lysis buffer $(1 \times$ PLB; $100 \mu \mathrm{L})$ was added, and the culture plate was placed on an orbital shaker with a shaking rate of $450 \mathrm{rpm}$ for $20 \mathrm{~min}$ at room temperature. Luciferase activity was determined according to the supplier's protocol by using cell lysate ( $30 \mu \mathrm{L})$ and an infinite M200 reader (Tecan, Männedorf, Switzerland).

\section{Acknowledgements}

J.S.H. gratefully acknowledges the Volkswagenstiftung for funding a Lichtenberg-Professorship. D.P.N.G. thanks the Portuguese Science Foundation (Fundaçao para a Ciência e a TecnologiaFCT-Portugal) SFRH/BD/12414/2003 (D.P.N.G.) for a studentship.

Keywords: gene expression • G quadruplexes RNA recognition - RNA structures $\cdot$ RNA switches

[1] C. C. Mello, D. Conte, Jr., Nature 2004, 431, 338.

[2] G. Meister, T. Tuschl, Nature 2004, 431, 343.

[3] Y. Lee, J. Han, K. H. Yeom, H. Jin, V. N. Kim, Cold Spring Harbor Symp. Quant. Biol. 2006, 71, 51.

[4] E. Lund, S. Guttinger, A. Calado, J. E. Dahlberg, U. Kutay, Science 2004, 303, 95.

[5] I. J. Macrae, K. Zhou, F. Li, A. Repic, A. N. Brooks, W. Z. Cande, P. D. Adams, J. A. Doudna, Science 2006, 311, 195.

[6] E. Lund, J. E. Dahlberg, Cold Spring Harbor Symp. Quant. Biol. 2006, 71, 59.

[7] C. Matranga, Y. Tomari, C. Shin, D. P. Bartel, P. D. Zamore, Cell 2005, 123, 607.

[8] D. S. Schwarz, G. Hutvagner, T. Du, Z. Xu, N. Aronin, P. D. Zamore, Cell 2003, 115, 199.

[9] G. Hutvagner, M. J. Simard, Nat. Rev. Mol. Cell Biol. 2008, 9, 22.

[10] D. V. Irvine, M. Zaratiegui, N. H. Tolia, D. B. Goto, D. H. Chitwood, M. W. Vaughn, L. Joshua-Tor, R. A. Martienssen, Science 2006, 313, 1134.

[11] S. M. Elbashir, J. Harborth, W. Lendeckel, A. Yalcin, K. Weber, T. Tuschl, Nature 2001, 411, 494.

[12] K. Chang, S. J. Elledge, G. J. Hannon, Nat. Methods 2006, 3, 707.

[13] J. R. Thomas, X. Liu, P. J. Hergenrother, J. Am. Chem. Soc. 2005, 127, 12434.

[14] K. Moehle, Z. Athanassiou, K. Patora, A. Davidson, G. Varani, J. A. Robinson, Angew. Chem. 2007, 119, 9260-9264; Angew. Chem. Int. Ed. 2007, 46, 9101.

[15] B. P. Davies, C. Arenz, Angew. Chem. 2006, 118, 5676; Angew. Chem. Int. Ed. 2006, 45, 5550.

[16] F. J. Isaacs, D. J. Dwyer, J. J. Collins, Nat. Biotechnol. 2006, 24, 545.

[17] M. N. Win, C. D. Smolke, Biotechnol. Genet. Eng. Rev. 2007, 24, 311.

[18] M. Wieland, J. S. Hartig, ChemBioChem 2008, 9, 1873.

[19] M. Famulok, J. S. Hartig, G. Mayer, Chem. Rev. 2007, 107, 3715. 
[20] T. S. Bayer, C. D. Smolke, Nat. Biotechnol. 2005, 23, 337.

[21] M. N. Win, C. D. Smolke, Proc. Natl. Acad. Sci. USA 2007, 104, 14283.

[22] M. Wieland, J. S. Hartig, Angew. Chem. 2008, 120, 2643; Angew. Chem. Int. Ed. 2008, 47, 2604.

[23] C. I. An, V. B. Trinh, Y. Yokobayashi, Rna 2006, 12, 710

[24] D. J. Patel, A. T. Phan, V. Kuryavyi, Nucleic Acids Res. 2007, 35, 7429.

[25] D. Monchaud, M. P. Teulade-Fichou, Org. Biomol. Chem. 2008, 6, 627.

[26] A. De Cian, L. Lacroix, C. Douarre, N. Temime-Smaali, C. Trentesaux, J.-F. Riou, J.-L. Mergny, Biochimie 2008, 90, 131.

[27] M. Wieland, J. S. Hartig, Angew. Chem. 2006, 118, 6007; Angew. Chem. Int. Ed. 2006, 45, 5875.

[28] A. Joachimi, G. Mayer, J. S. Hartig, J. Am. Chem. Soc. 2007, 129, 3036

[29] M. Wieland, J. S. Hartig, Chem. Biol. 2007, 14, 757.

[30] S. Burge, G. N. Parkinson, P. Hazel, A. K. Todd, S. Neidle, Nucleic Acids Res. 2006, 34, 5402.

[31] T. R. Cech, Cell 2004, 116, 273.

[32] H. Arthanari, P. H. Bolton, Chem. Biol. 2001, 8, 221.

[33] R. H. Shafer, I. Smirnov, Biopolymers 2000, 56, 209.

[34] J. Eddy, N. Maizels, Nucleic Acids Res. 2006, 34, 3887.

[35] S. Kumari, A. Bugaut, J. L. Huppert, S. Balasubramanian, Nat. Chem. Biol. 2007, 3, 218.

[36] R. Kostadinov, N. Malhotra, M. Viotti, R. Shine, L. D'Antonio, P. Bagga, Nucleic Acids Res. 2006, 34, D119.

[37] L. R. Kelland, Eur. J. Cancer 2005, 41, 971.

[38] A. Siddiqui-Jain, C. L. Grand, D. J. Bearss, L. H. Hurley, Proc. Natl. Acad. Sci. USA 2002, 99, 11593

[39] A. Rangan, O. Y. Fedoroff, L. H. Hurley, J. Biol. Chem. 2001, 276, 4640

[40] C. Granotier, G. Pennarun, L. Riou, F. Hoffschir, L. R. Gauthier, A. De Cian, D. Gomez, E. Mandine, J. F. Riou, J. L. Mergny, P. Mailliet, B. Dutrillaux, F. D. Boussin, Nucleic Acids Res. 2005, 33, 4182.

[41] J.-M. Zhou, X.-F. Zhu, Y.-J. Lu, R. Deng, Z.-S. Huang, Y.-P. Mei, Y. Wang, W.-L. Huang, Z.-C. Liu, L.-Q. Gu, Y.-X. Zeng, Oncogene 2006, 25, 503.

[42] A. M. Burger, F. Dai, C. M. Schultes, A.P. Reszka, M. J. Moore, J. A. Double, S. Neidle, Cancer Res. 2005, 65, 1489.

[43] A. De Cian, E. DeLemos, J.-L. Mergny, M.-P. Teulade-Fichou, D. Monchaud, J. Am. Chem. Soc. 2007, 129, 1856.
[44] D. P. N. Gonçalves, R. Rodriguez, S. Balasubramanian, J. K. M. Sanders, Chem. Commun. (Cambridge) 2006, 4685.

[45] Z. A. Waller, P. S. Shirude, R. Rodriguez, S. Balasubramanian, Chem. Commun. (Cambridge) 2008, 1467.

[46] A. Bugaut, K. Jantos, J. L. Wietor, R. Rodriguez, J. K. M. Sanders, S. Balasubramanian, Angew. Chem. 2008, 120, 2717-2720; Angew. Chem. Int Ed. 2008, 47, 2677.

[47] A. De Cian, J. L. Mergny, Nucleic Acids Res. 2007, 35, 2483.

[48] H. Han, C. L. Cliff, L. H. Hurley, Biochemistry 1999, 38, 6981.

[49] P. J. Perry, S. M. Gowan, A. P. Reszka, P. Polucci, T. C. Jenkins, L. R. Kelland, S. Neidle, J. Med. Chem. 1998, 41, 3253.

[50] S. Venitt, C. Crofton-Sleigh, M. Agbandje, T. C. Jenkins, S. Neidle, J. Med. Chem. 1998, 41, 3748.

[51] R. J. Harrison, S. M. Gowan, L. R. Kelland, S. Neidle, Bioorg. Med. Chem. Lett. 1999, 9, 2463.

[52] M. A. Read, A. A. Wood, J. R. Harrison, S. M. Gowan, L. R. Kelland, H. S. Dosanjh, S. Neidle, J. Med. Chem. 1999, 42, 4538.

[53] H. Han, D. R. Langley, A. Rangan, L. H. Hurley, J. Am. Chem. Soc. 2001, $123,8902$.

[54] E. Izbicka, R. T. Wheelhouse, E. Raymond, K. K. Davidson, R. A. Lawrence, D. Sun, B. E. Windle, L. H. Hurley, D. D. Von Hoff, Cancer Res. 1999, 59, 639.

[55] H. Arthanari, S. Basu, T. L. Kawano, P. H. Bolton, Nucleic Acids Res. 1998, 26, 3724.

[56] D. P. N. Gonçalves, S. Ladame, S. Balasubramanian, J. K. M. Sanders, Org. Biomol. Chem. 2006, 4, 3337.

[57] J. Ren, J. B. Chaires, Biochemistry 1999, 38, 16067.

[58] C. F. Tang, R. H. Shafer, J. Am. Chem. Soc. 2006, 128, 5966.

[59] I. J. MacRae, K. Zhou, J. A. Doudna, Nat. Struct. Mol. Biol. 2007, 14, 934.

[60] S. Y. Rha, E. Izbicka, R. Lawrence, K. Davidson, D. Sun, M. P. Moyer, G. D. Roodman, L. Hurley, D. Von Hoff, Clin. Cancer Res. 2000, 6, 987.

[61] C. L. Grand, H. Han, R. M. Munoz, S. Weitman, D. D. Von Hoff, L. H. Hurley, D. J. Bearss, Mol. Cancer Ther. 2002, 1, 565. 